\title{
AVALIAÇÃO DE DESASTRES NO RIO GRANDE DO SULASSOCIADOS A COMPLEXOS CONVECTIVOS DE MESOESCALA
}

\section{Evaluation of Hazards in Rio Grande do Sul, Brazil, Associated with Mesoscale Convective Complexes}

Denilson Ribeiro Viana Geógrafo pela Universidade Federal do Rio Grande do Sul Mestre em Sensoriamento Remoto pelo Instituto Nacional de Pesquisas Espaciais

São José dos Campos/SP - Brasil ribeiro.denilson@gmail.com

Francisco Eliseu Aquino

Prof. Assistente do Departamento de Geografia - Universidade Federal do Rio Grande do Sul Núcleo de Pesquisas Antárticas e Climáticas, NOTOS - Laboratório de Climatologia Porto Alegre/RS - Brasil francisco.aquino@ufrgs.br

Viviana Aguilar Muñoz

Engenheira Topógrafa pela Universidad del Valle - Cali, Colômbia Mestre em Sensoriamento Remoto pelo Instituto Nacional de Pesquisas Espaciais Assistente de pesquisa da Corporação Observatório Sismológico do Sul Ocidental, OSSO Cali, Valle del Cauca - Colômbia viaguila@gmail.com

Artigo recebido para publicação em 17/01/09 e aceito para publicação em 06/07/09

RESUMO: Os Complexos Convectivos de Mesoescala (CCM) são aglomerados organizados de nuvens convectivas, que atingem com freqüência a Região Sul do Brasil e produzem uma grande variedade de desastres. Este trabalho identificou os CCMs ocorridos entre outubro e dezembro de 2003 no Rio Grande do Sul (RS) e avaliou os desastres associados aos eventos. A identificação dos CCMs foi feita através do aplicativo ForTraCC - DSA/INPE. O levantamento das ocorrências de desastres foi realizado a partir do cruzamento dos dias de eventos de CCM com as informações da Defesa Civil do RS (DCRS) e do Jornal Correio do Povo. Foram identificados 22 eventos de CCM no período, responsáveis por 42 episódios de vendaval, 21 de enxurrada, 14 de enchente, cinco de granizo associado à vendaval, três de granizo, dois de alagamento, dois de inundação e um deslizamento, totalizando 90 ocorrências. Houve registro de seis mortes no RS e cinco em Santo Tomé na Argentina, fronteira com o Estado. Mais de 16.500 pessoas foram atingidas e cerca de $60 \%$ dos municípios que comunicaram desastres à DCRS decretaram situação de emergência. Em média, a cada quatro eventos de CCM, três produziram algum tipo de desastre no RS.

Palavras-chave: Complexos Convectivos de Mesoescala. Rio Grande do Sul. Desastres.

ABSTRACT: Mesoscale Convective Complexes (CCM) are organized clusters of convective clouds, which often arrive at Southern Region of Brazil and produce a variety of hazards. This study evaluated hazards 
associated with CCM events in the Rio Grande do Sul (RS) State, the southernmost of Brazil, between October and December of 2003. The detection of CCM was done using ForTraCC application DSA/INPE. Data coming from Defesa Civil do RS (DCRS), the RS civil defense organization, and from the archives of Jornal Correio do Povo, a local newspaper, served as source material to evaluate the hazards on the CCM event occurring days. During the period, 22 CCM events were identified over the RS. Based on DCRS reports, 90 incidents were associated with CCM events: strong gales and storms (42), flash floods (21), stream overflows (14), hail and strong gales (5), hail storms (3), swamping (2), floods (2) and earth flows (1). These CCM events caused the death of eleven people, of which six were in RS and the five in San Tomé, on the Argentinean side of the boarder. More than 16,500 people were affected by hazards caused by CCM in the RS. Approximately $60 \%$ of the municipal districts affected requested emergency situation. On average, three to four events produce some type of hazard.

Keywords: Mesoscale Convective Complexes. Rio Grande do Sul. Natural hazards.

\section{INTRODUÇÃO}

A ocorrência de fenômenos meteorológicos severos constitui-se em motivo de preocupação para a sociedade, pois oferece alto potencial de destruição, perdas de vidas humanas e bens materiais, abalando direta ou indiretamente sua economia. Granizo, rajadas de vento, tempestades elétricas, inundações e tornados são exemplos destas ocorrências, podendo causar sérios danos em moradias, equipamentos públicos, infra-estrutura urbana e rural, afetando principalmente a população mais vulnerável (setores socialmente mais desprotegidos). Estes episódios acabam, por vezes, agravando a situação de pessoas que dispõem de poucos recursos financeiros e, em muitas situações, estas acabam perdendo os poucos bens de que dispõem.

Os episódios de desastres associados a condições meteorológicas extremas são freqüentes no RS, tanto no verão, quanto nas estações de transição (primavera e outono). Esse período coincide com a temporada de atuação de sistemas convectivos no Estado. Abdoulaev et al. (1996) mostraram que esses sistemas causam, no mínimo, treze desastres por ano no RS.

A partir da década de 1980, com o avanço da tecnologia dos sensores a bordo dos satélites meteorológicos, foi possível compreender melhor as características de formação, desenvolvimento e trajetórias dos sistemas convectivos. Com base na observação de sucessivas imagens de satélite, uma classe especial desses sistemas foi identificada, tendo sido denominada Complexos Convectivos de Mesoescala (CCM). Maddox (1980) observou que esses sistemas possuíam formato aproximadamente circular e desenvolviam-se rapidamente, normalmente entre seis e doze horas.

Os CCMs foram reconhecidos inicialmente na região central dos Estados Unidos. No entanto, muitos estudos surgiram em várias regiões do planeta relatando a ocorrência desses sistemas, inclusive em latitudes médias na América do Sul (AS). Ainda hoje, apesar dos avanços na meteorologia, os CCMs são de difícil previsibilidade, já que os eventos não estão associados a nenhum tipo de sistema meteorológico, como frentes frias ou linhas de instabilidade. Sua gênese e desenvolvimento são resultantes da umidade disponível na atmosfera, em baixos níveis; da circulação do ar superior, em altos níveis; e da circulação local (MADDOX, 1980).

Este trabalho tem como objetivos identificar os CCMs que atingiram o RS entre outubro e dezem- 
bro de 2003 e avaliar os prejuízos materiais e perdas humanas ocasionados pelos eventos. O estudo visa contribuir para o entendimento dos mecanismos de formação e desenvolvimento dos CCMs, seu possível impacto na sociedade e suas implicações no planejamento territorial e na prevenção de desastres. A escassez de trabalhos sobre a influência e transtornos causados por CCM na Região Sul do Brasil foi o fator que motivou a realização desta pesquisa.

\section{REFERENCIAL TEÓRICO}

\subsection{Caracterização dos Complexos Convectivos de Mesoescala}

Nos Estados Unidos, a partir da década de 1980, uma classe particular de sistemas convectivos foi observada por Maddox (1980). O autor avaliou um número significativo de desastres na região central dos EUA, em condições sinóticas que não estavam relacionadas à passagem de frentes frias ou a atuação de linhas de instabilidades. Essas condições foram descritas sumariamente por Maddox (1980), que associou a gênese desses eventos ao escoamento vale-montanha, na borda leste das montanhas Rochosas; a entrada de ar úmido oriundo do golfo do México, através de jatos de baixos níveis; e ao posicionamento do jato de altos níveis.

Os CCMs caracterizam-se como uma junção de células convectivas individuais (nuvens Cumulunimbus), cobertos por uma extensa camada de nuvens Cirrus, com formato quase circular e um rápido crescimento entre seis e doze horas (MADDOX, 1980; MADDOX, 1983). Na AS, os CCM se desenvolvem normalmente entre as latitudes $20^{\circ}$ e $30^{\circ} \mathrm{S}$, nas regiões norte da Argentina, Paraguai e Bolívia, e atingem com freqüência a Região Sul do Brasil (VELASCO; FRITSCH, 1987; SCOLAR; FIGUEIREDO, 1990). Ocorrem, sobretudo, na metade quente do ano, e produzem grande variedade de fenômenos meteorológicos adversos, tais como tornados, granizo, fortes rajadas de vento, enchentes, inundações e tempestades elétricas (MADDOX, 1980; MADDOX, 1983).

A partir da análise de dezenas de casos de sis- temas convectivos, através de imagens de satélite no canal infravermelho, Maddox (1980) introduziu um modelo conceitual para a classificação desses sistemas, baseado nas características físicas dos topos nebulosos, obtidas através de técnicas de realce. Os CCMs, para serem classificados como tal, devem satisfazer a restrição em relação à excentricidade (excluindo sistemas lineares do tipo linhas de instabilidade) e aos limiares de temperatura de topo de nuvem.

Após a proposição estabelecida por Maddox (1980) para classificação dos CCMs, vários estudos seguiram nos EUA e em outras regiões do planeta, buscando caracterizar tanto a ocorrência dos eventos, quanto a de fenômenos extremos associados. Severos temporais - tornados, ventos intensos e granizo -, bem como chuva torrencial e inundações foram observadas nos casos analisados. Segundo Maddox (1983), um em cada cinco eventos produziu feridos ou mortos.

No que diz respeito à precipitação associada, Fritsch et al. (1986) estudando 74 casos de CCM ocorridos entre 1982 e 1983, observaram que os eventos contribuíram entre $20 \%$ e $50 \%$ do total anual de precipitação na região da Planície Central dos EUA, sendo que, entre junho e agosto, a precipitação é particularmente dominada por CCM. McAnelly e Cotton (1989) analisaram sete anos de informações de CCM - nos meses de junho a agosto, entre 1977 e 1983 -, e concluíram que, em 122 casos analisados, a participação de CCM na precipitação chegou a valores da ordem de $45 \%$.

$\mathrm{Na}$ AS, Satyamurty et al. (1998) salientam que os setores oeste e noroeste do RS são locais propícios à formação e desenvolvimento de CCM. A distribuição da precipitação observada no verão e nas estações de transição sugere que os CCM sejam um dos mecanismos responsáveis pelos acumulados registrados em toda a Região Sul, neste período. No RS, destaca-se a região noroeste, onde os acumulados de precipitação são significativos nessas estações (SEVERO; GAN, 2004). Viana et al. (2009) analisaram a precipitação associada aos CCMs entre outubro e dezembro de 2003 no RS, e concluíram que aproximadamente dois terços do volume de chuvas registrado foi oriundo dos eventos. 
Velasco e Fritsch (1987) avaliaram a ocorrência, freqüência e características dos CCMs em latitudes médias na AS. O estudo proporcionou uma base sinótica para promover o entendimento de sistemas atmosféricos em latitudes médias, durante os meses quentes do ano. Segundo os autores, entre 1981 e 1983 foram identificados 78 eventos, que causaram, além de precipitações significativas, fenômenos como rajadas de vento, tornados e enchentes; ocasionando prejuízos materiais e perdas de vidas humanas.

Guedes et al. (1994) analisaram as trajetórias dos sistemas convectivos com tempo superior a seis horas, sobre a AS, no período de Jul/1987 a Jun/1988. Os autores concluíram que no verão, sobre a região Sul e Sudeste do Brasil, esses sistemas se deslocam de sudoeste para nordeste, percorrendo uma distância maior, quando comparada com o período de inverno. Machado et al. (1998) apontaram que as trajetórias de sistemas convectivos de latitudes médias na AS, no verão e outono, são similares as obtidas por Guedes e Silva Dias (1984) e por Velasco e Fristch (1987). A FIG. 1 ilustra um evento de CCM identificado no presente trabalho, localizado na fronteira entre o Brasil e a Argentina em 20/12/2003.

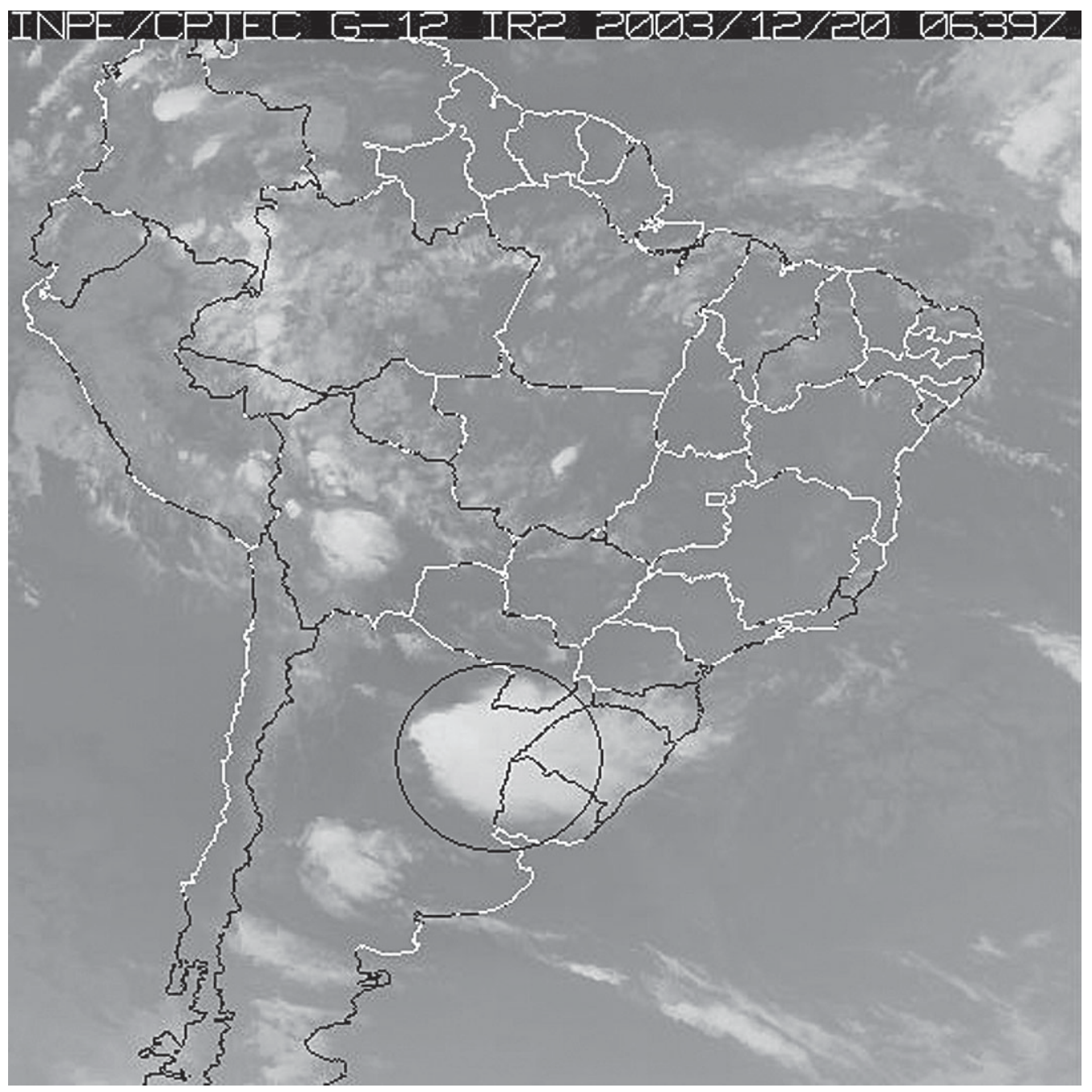

FIGURA 1. Imagem do satélite GOES-12 (canal infravermelho) no dia 20/12/2003 mostrando um evento de CCM sobre o norte da Argentina e região oeste do RS. Fonte: CPTEC/INPE (2004). 
Em latitudes médias na AS, os CCMs são sistemas predominantemente noturnos e seu horário de maior atividade é durante a madrugada. As primeiras células convectivas, que ainda precedem o início do fenômeno, podem ocorrer tanto no início da tarde, como da noite. A duração média dos CCMs varia entre 10 e 20 horas (SILVA DIAS, 1987). Machado et al. (1994) examinaram o ciclo de vida de sistemas convectivos sobre a AS e encontraram um tempo de vida médio de 15 horas para latitudes médias no verão.

O transporte de calor e umidade das latitudes baixas para os subtrópicos representam o fator primordial para a formação e desenvolvimento dos CCMs. Marengo et al. (2004) apontaram que uma das características das circulações na AS é a composição durante o verão de um Jato de Baixo Nível (JBN), mostrando o aumento do transporte meridional de umidade em baixos níveis, oriundo do Equador. Os JBN ocorrem ao longo do ano, trazendo massas de ar úmidas da Amazônia para o sul do Brasil e norte da Argentina, porém são mais freqüentes no verão, sendo seu ciclo mais intenso durante a madrugada, entre as 06:00 e 12:00 UTC. Paegle (1987) e Torres e Nicollini (2002), analisando a relação dos JBN com os sistemas convectivos sobre a AS, mostraram que o jato a leste dos Andes, produziu um ambiente favorável para a organização de convecção profunda. Nogués-Peagle e Berbery (2000) observaram na AS uma extensa área de convecção predominantemente noturna durante a primavera e verão, situação essa, capaz de alterar sistematicamente o elemento básico de precipitação no continente sul-americano.

\subsection{Conceito de Desastres}

Existem diversas definições para desastres, sendo que estas dependem do enfoque (físico, infra-estrutura e social) e da natureza das ocorrências (geológica, climática, antrópica, etc.). Estas definições geralmente referem-se às conseqüências e não as causas dos fenômenos. Aysan e Oliver (1987, p. 66), com base na experiência em danos provocados por terremotos, definem desastres como "a interação entre um fenômeno geofísico extremo e uma condição de vulnerabilidade, que se traduz em perdas econômicas e humanas, em uma escala totalmente fora das capacidades e recursos da administração local". Outras definições, como as resumidas por Wijkman e Timberlake (1985), incluem o número de mortos e feridos, bem como o valor das perdas materiais. Outros autores consideram o caráter imprevisível dos fenômenos e o despreparo dos governos para lidar com as conseqüências sociais e políticas provocadas (CUNY, 1983).

No Brasil, Castro (1998, p. 82) define desastres como "o resultado de eventos adversos, naturais ou provocados pelo homem, sobre um ecossistema (vulnerável), causando danos humanos, materiais e/ou ambientais e conseqüentes prejuízos econômicos e sociais." Os desastres são normalmente súbitos e inesperados, de uma gravidade e magnitude capaz de produzir danos materiais e humanos (inclusive com mortos e feridos) e prejuízos sócio-econômicos (KOBIYAMA et al., 2006).

A ocorrência de desastres está ligada não somente à susceptibilidade, devido às características geoambientais, mas também à vulnerabilidade do sistema econômico-social-político-cultural sob impacto (ALCÁNTARA-AYALA, 2002). Por vulnerabilidade entende-se a incapacidade de uma comunidade de "absorver" os efeitos de determinada alteração em seu meio, ou seja, a incapacidade de adaptar-se às modificações (WILCHES-CHAUX, 1993). A vulnerabilidade determina a intensidade dos danos que produz a ocorrência efetiva de um risco sobre essa comunidade. $\mathrm{O}$ risco trata-se da probabilidade da "perda", enquanto o desastre é a perda concretizada (MASKREY, 1998).

As pesquisas na temática de desastres têm se desenvolvido a partir de vários enfoques, incluindo as ciências naturais, sociais e aplicadas. Segundo Maskrey (1998), o principal aporte das ciências naturais foi o desenvolvimento dos estudos da ameaça e o das ciências sociais, por sua vez, foram os estudos de vulnerabilidade da população. A integração desses conceitos, em um modelo matemático, é o aporte das ciências aplicadas, onde o risco é considerado como uma função que relaciona esses conceitos (ameaça e vulnerabilidade). 
Os desastres podem ser classificados a partir de três aspectos, conforme Castro (1999):

a) Intensidade: divididos em quatro níveis (pequeno, médio, grande e muito significativo), a partir dos prejuízos avaliados;

b) Evolução: refere-se à velocidade do evento, classificada em súbitos (inundações, vendavais e tornados), graduais (inundações lentas e secas) e somação dos efeitos parciais;

c) Origem: classificados em naturais (fenômenos naturais extremos, que independem da ação humana), antrópicos (causados pela ação ou omissão humana) e mistos (associados às ações ou omissões humanas, que contribuem para intensificar os desastres).

As ocorrências desencadeadas por fenômenos meteorológicos severos e causadoras de desastres são descritas conforme publicação organizada por Kobiyama et al. (2006):

a) Inundação: trata-se do aumento do nível dos rios além da sua vazão normal, ocorrendo o transbordamento de suas águas sobre a planície de inundação. A Defesa Civil classifica as inundações em função da magnitude (excepcionais, de grande magnitude, normais ou regulares e de pequena magnitude) e em função do padrão evolutivo (inundações graduais, inundações bruscas, alagamentos e inundações litorâneas) (CASTRO, 2003).

b) Enchente: quando não ocorre o transbordamento, apesar do rio ficar praticamente cheio. Os termos "enchente" e "inundação" são freqüentemente empregados como sinônimos, no entanto devem ser usados com diferenciação.

c) Enxurrada: inundação brusca devido à chuvas intensas e concentradas, principalmente em regiões de relevo acidentado. d) Escorregamento ou deslizamento: movimento coletivo de massa e/ou material sólido encosta abaixo, como solos, rochas e vegetação, sob a influência direta da gravidade (SELBY, 1993). Estes movimentos podem ocorrer principalmente com elevados volumes de precipitação e/ou terremotos. Tanto chuvas intensas de curta duração, quanto de longa duração (chuvas contínuas), fornecem condições propícias para a diminuição da resistência do solo, atuando como um dos principais agentes deflagradores de movimentos de encostas, especialmente em ambientes tropicais úmidos (GUIDICINI; IWASA, 1976).

e) Granizo: precipitação de gelo, em forma esférica ou irregular, apresentando geralmente um diâmetro de 5 mm (GLICKMAN, 2000). As condições que propiciam a formação de granizo acontecem na parte superior de nuvens do tipo Cumulunimbus, que possuem temperaturas muito baixas. Em função da união de gotas congeladas, o granizo cresce rapidamente. Além disso, as gotas congeladas movimentamse com as correntes subsidentes e ascendentes, chocando-se com gotas de água mais frias até alcançarem as dimensões de queda (KULICOV; RUDNEV, 1980; KNIGHT; KNIGHT, 2001).

f) Vendaval: deslocamento intenso de ar na superfície terrestre devido, principalmente, às diferenças no gradiente de pressão atmosférica, aos movimentos descendentes e ascendentes do ar e a rugosidade do terreno (VIANELLO; ALVES, 1992). O relevo também pode contribuir significativamente para a intensificação dos ventos. As variações bruscas na velocidade do vento denominam-se rajadas, as quais, normalmente são acompanhadas por mudanças bruscas na direção. Estas rajadas também podem variar consideravelmente em virtude da rugosidade do terreno, seja ela natural (colinas, morros, vales, etc.) ou construída (casas, prédios, etc.) (BRYANT, 1991). 


\section{DADOS E METODOLOGIA}

\subsection{Identificação dos Complexos Convectivos de Mesoescala}

Para a classificação dos eventos de CCM no período de outubro a dezembro de 2003 foi utilizado o aplicativo ForTraCC (Forecast and Tracking of Active Convective Cells) com base nos critérios estabelecidos por Maddox (1980). O ForTraCC foi desenvolvido pela equipe da Divisão de Satélites Ambientais (DSA) do Instituto Nacional de Pesquisas Espaciais (INPE), e concentra-se na determinação de trajetórias e ciclo de vida de sistemas convectivos, através da utilização de imagens do satélite GOES (Geostationary Operational Environmental Satellites). O programa utiliza os limiares de temperatura de topos nebulosos de $235 \mathrm{~K}\left(-38^{\circ} \mathrm{C}\right)$ para definir o sistema, de $210 \mathrm{~K}$ ($63^{\circ} \mathrm{C}$ ) para definir as células convectivas e ainda de $250 \mathrm{~K}\left(-23^{\circ} \mathrm{C}\right)$ para detecção precoce de sistemas convectivos.

Cabe destacar que, para a realização desse trabalho, foi solicitado à equipe da DSA-INPE o reprocessamento do ForTraCC no período, alterando os limiares de temperatura de topos nebulosos. Os limiares para rastreamento de sistemas convectivos passaram de $235 \mathrm{~K}\left(-38^{\circ} \mathrm{C}\right)$ para $241 \mathrm{~K}\left(-32^{\circ} \mathrm{C}\right)$ (limiar quente) e de $210 \mathrm{~K}\left(-63^{\circ} \mathrm{C}\right)$ para $221 \mathrm{~K}\left(-52^{\circ}\right)$ (limiar frio). A partir da alteração desses limiares foi possível detectar sistemas convectivos com características de $\mathrm{CCM}$, e avaliar os demais parâmetros físicos. Isso permitiu a identificação precisa dos eventos e das respectivas datas e locais de ocorrência, de acordo com a metodologia proposta por Maddox (1980) para classificação do fenômeno.

Segundo Macedo et al. (2004), o método de identificação de um mesmo sistema no tempo (t e $t+\Delta t$ ), é obtido através do emprego do critério de máxima superposição da área dos sistemas em sucessivas imagens de satélite. Adota-se um número mínimo de pixels para considerar a continuidade do sistema convectivo (150 pixels, o que corresponde a aproximadamente $2.400 \mathrm{~km}^{2}$ ) para $\Delta \mathrm{t}=30$ minutos. Se a superposição for menor, o sistema não é considerado como o mes- mo sistema no tempo anterior.

\subsection{Avaliação de Desastres}

A avaliação dos desastres associados aos eventos de CCM foi verificada a partir dos dados da Defesa Civil do Rio Grande do Sul (DCRS) e do Jornal Correio do Povo (JCP), ambos disponíveis na internet. O período para seleção dos desastres abrange os dias compreendidos entre o início e o término de cada um dos eventos de CCM (TAB. 1, coluna "Dias de ocorrência de desastres"). Foram contabilizados somente os desastres ocorridos nos locais onde a área da fração convectiva dos sistemas (nuvens Cumulonimbus) tenha atingido o RS em algum momento do seu ciclo de vida. Para isso, foram utilizadas imagens do satélite GOES-12 e Meteosat, no canal Infravermelho, realçadas de acordo com a temperatura de topos de nuvens, em intervalos de tempo de meia hora. Ao todo foram reunidas cerca de 9.700 imagens, que permitiram acompanhar a evolução e a trajetória dos CCMs.

As ocorrências desencadeadas por fenômenos meteorológicos adversos e causadores de desastres são classificadas segundo a DCRS (2006) em: alagamentos, deslizamentos, enchentes, enxurradas, granizo, inundações, tornados e vendavais. Para regionalização das ocorrências, a DCRS divide o Estado em cinco coordenadorias Regionais de Defesa Civil (REDECs): Metropolitana, Passo Fundo, Santa Maria, Pelotas e Santo Ângelo. Esta regionalização foi utilizada na avaliação dos desastres verificados no período (FIG. 2).

Os desastres "pontuais", muitas vezes não comunicados à Defesa Civil, foram verificados a partir das notícias veiculadas no JCP. As ocorrências evolvem casos como destelhamentos de casas, falta de energia elétrica, queda de árvores e postes, entre outras. Dessa forma, foi possível elaborar um panorama geral das ocorrências de desastres associados aos CCMs, considerando os diferentes níveis de intensidade, apontados por Castro (1999). 


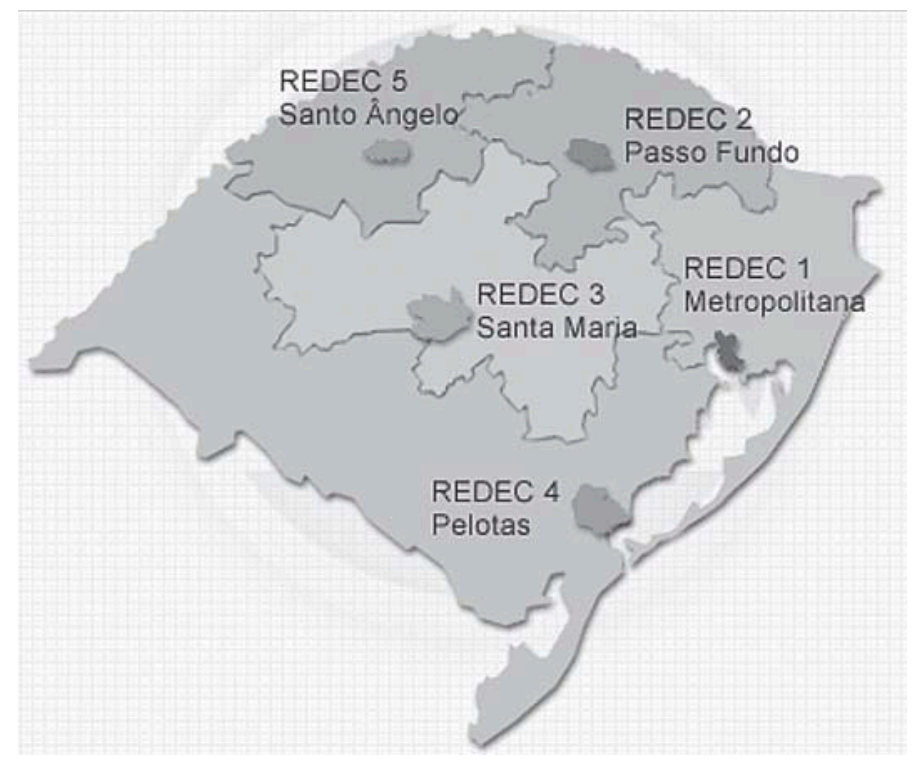

FIGURA 2. Coordenadorias Regionais de Defesa Civil do RS. Fonte: DCRS (2006).

\section{RESULTADOS E DISCUSSÕES}

\subsection{Identificação dos Eventos de CCM}

Foram identificados vinte e dois eventos de CCM no trimestre onde, desse total, sete ocorreram em outubro, sete em novembro e oito em dezembro. A
TAB. 1 descreve as principais características dos CCMs observados, tais como as datas de ocorrência, a duração, os dias utilizados para a avaliação de desastres e as características físicas (tamanhos A, B e excentricidade) no momento de máxima extensão. A FIG. 3 ilustra a localização do centro geométrico dos CCMs no momento de máxima extensão.

TABELA 1. Principais características dos CCMs no período de outubro a dezembro de 2003.

\begin{tabular}{|c|c|c|c|c|c|c|c|c|}
\hline $\begin{array}{l}\text { Cód. } \\
\text { Evento } \\
\text { CCM }\end{array}$ & $\begin{array}{l}\text { Data Hora } \\
\text { Início } \\
\text { CCM (HL) }\end{array}$ & $\begin{array}{l}\text { Data Hora } \\
\text { Max Ext } \\
\text { CCM (HL) }\end{array}$ & $\begin{array}{l}\text { Data Hora } \\
\text { Fim } \\
\text { CCM (HL) }\end{array}$ & $\begin{array}{c}\text { Duração } \\
\text { CCM } \\
\text { (horas) }\end{array}$ & $\begin{array}{c}\text { Dias de } \\
\text { ocorrência de } \\
\text { desastres }\end{array}$ & $\begin{array}{c}\text { Tam A } \\
\text { HorMaxExt } \\
\quad\left(\mathrm{km}^{2}\right)\end{array}$ & $\begin{array}{c}\text { Tam B } \\
\text { HorMaxExt } \\
\quad\left(\mathrm{km}^{2}\right)\end{array}$ & $\begin{array}{l}\text { Excentric } \\
\text { HorMaxExt }\end{array}$ \\
\hline A & $03 / 10 \quad 04: 45$ & $03 / 10 \quad 14: 45$ & $03 / 10 \quad 15: 45$ & $11: 00$ & $3 / 10$ & 479.344 & 147.840 & 0,8 \\
\hline B & $03 / 10 \quad 16: 15$ & $03 / 10 \quad 20: 45$ & $04 / 10 \quad 03: 15$ & $11: 00$ & 3 e $4 / 10$ & 674.064 & 85.840 & 0,7 \\
\hline $\mathrm{C}$ & $04 / 10 \quad 04: 15$ & $04 / 10 \quad 05: 45$ & $04 / 10 \quad 14: 15$ & $10: 00$ & $4 / 10$ & 766.928 & 280.256 & 0,7 \\
\hline $\mathrm{D}$ & $21 / 10 \quad 01: 15$ & $21 / 10 \quad 12: 45$ & $21 / 10 \quad 15: 15$ & $14: 00$ & $21 / 10$ & 683.536 & 143.136 & 0,8 \\
\hline $\mathrm{E}$ & $24 / 10 \quad 18: 15$ & $25 / 10 \quad 01: 15$ & $25 / 10 \quad 02: 45$ & $8: 30$ & 24 e $25 / 10$ & 589.840 & 256.864 & 0,9 \\
\hline $\mathrm{F}$ & $25 / 10 \quad 06: 45$ & $25 / 10 \quad 23: 15$ & $26 / 10 \quad 10: 45$ & 28:00 & 25 e $26 / 10$ & 2.209 .456 & 1.063 .216 & 1,0 \\
\hline G & $31 / 10 \quad 16: 15$ & $31 / 10 \quad 18: 45$ & $01 / 11 \quad 01: 15$ & $9: 00$ & $31 / 10$ e $01 / 11$ & 849.472 & 177.840 & 0,8 \\
\hline $\mathrm{H}$ & $10 / 11 \quad 20: 45$ & $11 / 11 \quad 06: 45$ & $11 / 11 \quad 21: 15$ & $24: 30$ & 10 e $11 / 11$ & 1.080 .064 & 460.032 & 0,8 \\
\hline I & $12 / 11 \quad 15: 45$ & $12 / 11 \quad 20: 45$ & $12 / 11 \quad 21: 45$ & $6: 00$ & $12 / 11$ & 953.136 & 415.616 & 0,7 \\
\hline $\mathrm{J}$ & $15 / 11 \quad 15: 45$ & $15 / 11 \quad 22: 15$ & $16 / 11 \quad 00: 45$ & 9:00 & 15 e $16 / 11$ & 342.528 & 159.312 & 0,7 \\
\hline $\mathrm{K}$ & $16 / 11 \quad 11: 15$ & $17 / 11 \quad 12: 15$ & $17 / 11 \quad 12: 15$ & $25: 00$ & 16 e $17 / 11$ & 2.122 .976 & 732.672 & 1,0 \\
\hline $\mathrm{L}$ & $17 / 11 \quad 14: 15$ & $17 / 11 \quad 18: 45$ & $17 / 11 \quad 21: 45$ & $7: 30$ & $17 / 11$ & 1.104 .016 & 548.000 & 0,7 \\
\hline M & $25 / 11 \quad 08: 45$ & $25 / 11 \quad 14: 45$ & $25 / 11 \quad 14: 45$ & $6: 00$ & $25 / 11$ & 651.696 & 279.680 & 1,0 \\
\hline $\mathrm{N}$ & $26 / 11 \quad 03: 45$ & $27 / 11 \quad 01: 45$ & $27 / 11 \quad 02: 45$ & $23: 00$ & 26 e $27 / 11$ & 2.055 .312 & 1.096 .048 & 0,7 \\
\hline $\mathrm{O}$ & $07 / 12 \quad 15: 45$ & $09 / 12 \quad 03: 45$ & 09/12 06:45 & $39: 00$ & 7 a $9 / 12$ & 3.427 .360 & 901.296 & 0,7 \\
\hline $\mathrm{P}$ & $12 / 12 \quad 20: 15$ & $13 / 12 \quad 12: 45$ & $13 / 12 \quad 15: 15$ & $19: 00$ & 12 e $13 / 12$ & 654.816 & 129.600 & 0,7 \\
\hline Q & $14 / 12 \quad 01: 45$ & $14 / 12 \quad 03: 45$ & $14 / 12 \quad 15: 15$ & $13: 30$ & $14 / 12$ & 342.576 & 160.992 & 0,7 \\
\hline $\mathrm{R}$ & $14 / 12 \quad 17: 45$ & $15 / 12 \quad 03: 45$ & $15 / 12 \quad 07: 15$ & $13: 30$ & 14 e $15 / 12$ & 330.208 & 221.936 & 0,7 \\
\hline $\mathrm{S}$ & $14 / 12 \quad 20: 15$ & $15 / 12 \quad 09: 15$ & $15 / 12 \quad 15: 15$ & 19:00 & 14 e $15 / 12$ & 840.768 & 159.104 & 0,7 \\
\hline $\mathrm{T}$ & $20 / 12 \quad 09: 45$ & $20 / 12 \quad 15: 45$ & $21 / 12 \quad 10: 45$ & $25: 00$ & 20 e $21 / 12$ & 1.076 .864 & 309.680 & 1,0 \\
\hline $\mathrm{U}$ & $21 / 12 \quad 08: 45$ & $22 / 12 \quad 08: 15$ & $22 / 12 \quad 08: 15$ & $23: 30$ & 21 e $22 / 12$ & 1.362 .112 & 769.296 & 0,7 \\
\hline V & $25 / 12 \quad 17: 45$ & $27 / 12 \quad 02: 45$ & $28 / 12 \quad 10: 45$ & $65: 00$ & 25 a $28 / 12$ & 1.387 .088 & 650.880 & 0,7 \\
\hline
\end{tabular}




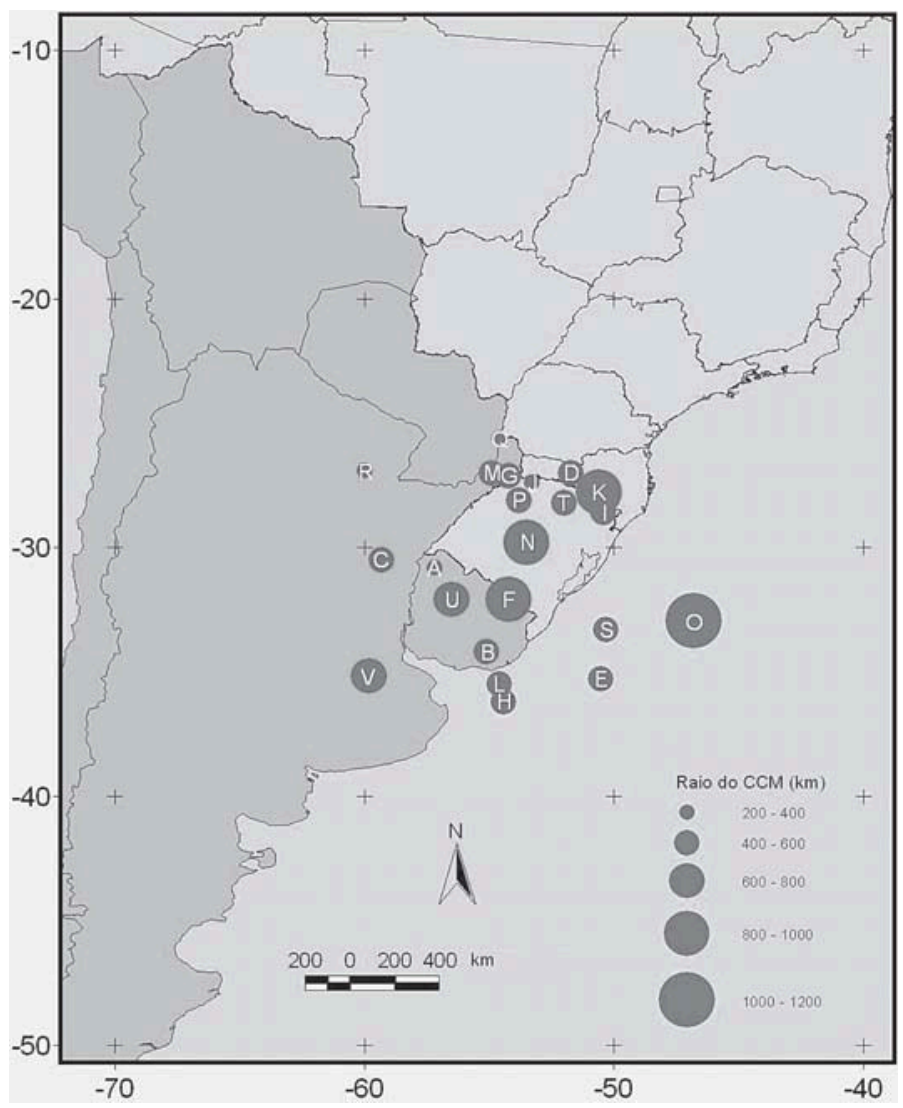

FIGURA 3. Localização dos CCM que atuaram sobre o RS no trimestre (*).

(*) as letras correspondem aos eventos listados na coluna 'Cód Evento CCM' na TAB. 1.

\subsection{Desastres associados a Complexos Convectivos de Mesoescala}

Foram verificadas 90 ocorrências desencadeadas por fenômenos meteorológicos associados aos CCMs durante o período, conforme o cruzamento entre os dias de ocorrência dos eventos e os episódios comunicados pelos municípios à DCRS. Esse número corresponde a $63 \%$ do total de ocorrências verificadas no trimestre, revelando que a participação dos CCMs foi de quase dois terços nos episódios de eventos extremos ocorridos no RS. A TAB. 2 apresenta as ocorrências comunicadas pelos municípios à DCRS.

TABELA 2. Ocorrências de fenômenos meteorológicos adversos associados aos CCMs

\begin{tabular}{|c|c|c|c|c|c|}
\hline Ocorrências & Out/2003 & Nov/2003 & Dez/2003 & Total & Participação \\
\hline Vendaval & 27 & 6 & 9 & 42 & $47 \%$ \\
\hline Enxurrada & 2 & - & 19 & 21 & $23 \%$ \\
\hline Enchente & 1 & - & 13 & 14 & $16 \%$ \\
\hline Granizo/Vendaval ${ }^{(1)}$ & 5 & - & - & 5 & $6 \%$ \\
\hline Granizo & - & 2 & 1 & 3 & $3 \%$ \\
\hline Alagamento & - & - & 2 & 2 & $2 \%$ \\
\hline Inundação & 1 & - & 1 & 2 & $2 \%$ \\
\hline Deslizamento & - & - & 1 & 1 & $1 \%$ \\
\hline Total & 36 & 8 & 46 & 90 & \\
\hline
\end{tabular}

Fonte: Defesa Civil (Rio Grande do Sul, 2006). ${ }^{(1)}$ Ocorrência simultânea dos dois eventos. 
O maior número de ocorrências foi de vendavais $(47 \%)$, seguidos de enxurradas $(23 \%)$, enchentes $(16 \%)$, granizo/vendaval $(6 \%)$, granizo $(3 \%)$, alagamentos $(2 \%)$, inundações $(2 \%)$ e deslizamento $(1 \%)$. Dezembro apresentou o maior número de episódios (46), seguido do mês de outubro (36). Novembro registrou um baixo número de eventos adversos (8), comparado com os demais meses. Destaca-se uma diferença nos tipos de ocorrências reportadas entre os meses de outubro e dezembro. Em outubro houve predomínio de episódios de vendavais e granizo, totalizando $89 \%$ das ocorrências. Já em dezembro, o maior número de registros esteve associado ao excessivo volume de precipitação (enxurradas, enchentes, inundações, alagamentos e deslizamentos) que corresponderam a $77 \%$ do total de ocorrências do mês. A FIG. 4 ilustra a participação dos episódios de eventos extremos associados aos CCMs no trimestre.

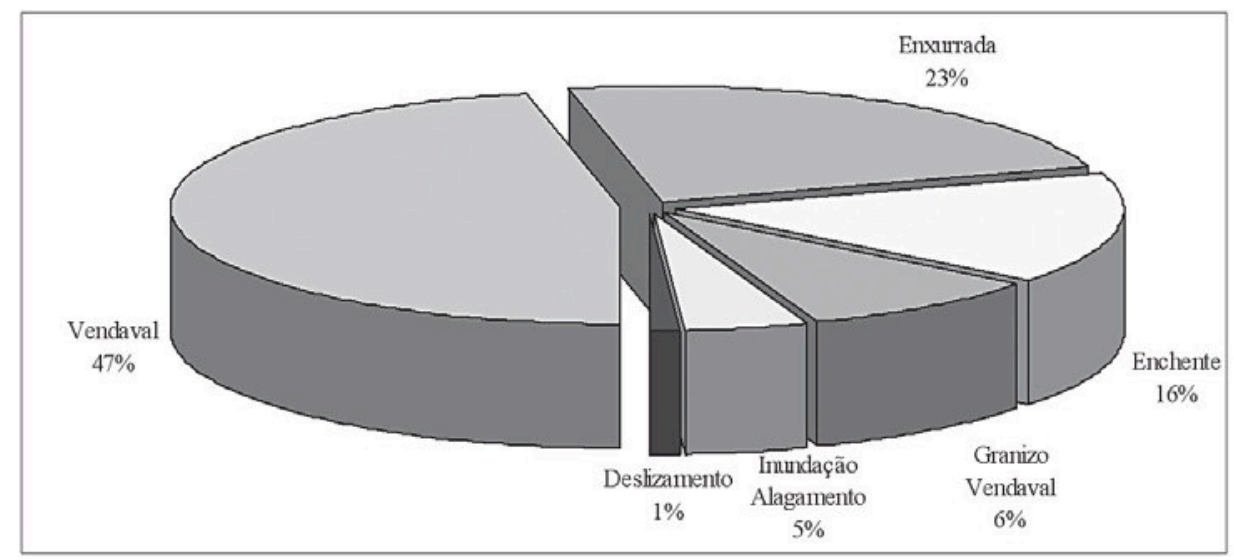

FIGURA 4. Episódios de eventos extremos associados aos CCMs no trimestre (\%).

\subsubsection{Outubro}

Os primeiros quatro eventos de CCMs observados nos dias 03,04 e 21/10 ( $A, B, C$ e $D$; TAB. 1$)$ não registraram episódios significativos de desastres, já que nenhuma ocorrência foi noticiada pelo JCP ou comunicada à DCRS. No entanto, os eventos $E$ e $F$ (TAB. 1), ocorridos entre os dias 24 e 26/10, causaram uma série de danos em grade parte do Estado, atingindo cerca de 5.000 pessoas. Fortes chuvas, rajadas de vento de até $100 \mathrm{~km} / \mathrm{h}$ e precipitações de granizo provocaram alagamentos e destruição em várias regiões. Segundo a DCRS (2006), 36 municípios comunicaram algum tipo de ocorrência, dois quais 24 decretaram situação de emergência. A região de Santo Ângelo foi a mais atingida com 58\% dos registros. Em 27 municípios houve episódios de vendavais. No interior, as cidades com os maiores danos foram Bagé, Passo Fundo, Palmeira das Missões e Guarani das Missões.

Na capital, Porto Alegre, atingida por dois temporais em menos de quatro horas, houve sérios alaga- mentos em diversos pontos. Foram relatados casos de falta de energia elétrica, problemas em semáforos, quedas de fios de alta tensão e de árvores, moradias destelhadas e inundadas pela água, resultando em várias famílias desabrigadas. Também houve falta de energia elétrica na região Metropolitana. Em Eldorado do Sul, o temporal destelhou inúmeras casas e alagou várias lojas na área comercial. Em Santa Maria, região central do Estado, um raio provocou blecaute de 30 minutos. Em Bagé houve destelhamento de centenas de casas em alguns bairros e duas pessoas ficaram feridas em razão de desabamentos.

O município de Passo Fundo registrou ventos de $91 \mathrm{~km} / \mathrm{h}$, onde 110 moradias foram danificadas. Segundo a DCRS (2006), o temporal resultou em residências inundadas, danos na rede elétrica e telefônica, queda de árvores, interrupção do trânsito, destruição de trechos de estradas, pontes, pontilhões e bueiros. Em Trindade do Sul, no Norte do Estado, houve o destelhamento e danos parciais em aproximadamente 550 casas. Várias residências foram totalmente 
destruídas, resultando em duas vítimas fatais. No interior do município de Vera Cruz, no Vale do Rio Pardo, uma mulher de 34 anos morreu vítima de descarga elétrica.

O CCM ocorrido entre 31/10 e 01/11/2003 (evento $G$; TAB. 1) trouxe mais uma vez prejuízos à capital, Porto Alegre. Foram registrados fortes ventos e precipitação intensa durante a madrugada. As rajadas de vento de até $78 \mathrm{~km} / \mathrm{h}$ causaram a queda de árvores e postes de energia, danificando veículos.

\subsubsection{Novembro}

Os desastres associados aos CCMs em novembro atingiram aproximadamente 1.200 pessoas no Estado. Comparado aos meses de outubro e dezembro, o número de ocorrências foi pequeno, representando cerca de $9 \%$ dos registros do trimestre. A região de Santo Ângelo mais uma vez foi a mais atingida, desta vez com $75 \%$ das ocorrências de eventos extremos. A maior parte dos registros foi de vendavais $(75 \%)$, seguido de precipitação de granizo (25\%).

O evento $I$ (TAB. 1), verificado na noite de 12/11 e madrugada do dia 13/11/2003, provocou uma série de prejuízos na fronteira oeste do RS. Segundo a DCRS, nos municípios de Palmitinho e Pinheirinho do Vale a precipitação de granizo danificou cerca de 230 residências, atingindo 550 pessoas. Ambos os municípios decretaram situação de emergência. O JCP (2006) relata outros estragos provocados por esse evento. Em Livramento, fronteira sul, duas casas foram destruídas e pelo menos 15 ficaram destelhadas. A maior parte da cidade ficou com o abastecimento de energia comprometido. Foram registrados ventos de até $90 \mathrm{~km} / \mathrm{h}$ que derrubaram muros, árvores e galhos sobre a rede elétrica. Áreas da zona rural e da vizinha cidade uruguaia de Rivera também foram atingidas pelo temporal. Em Rosário do Sul, o vento derrubou árvores e deixou parte da população sem energia elétrica.

Os CCMs ocorridos entre os dias 15 e 17/11 (eventos $J, K$ e $L$; TAB. 1) causaram uma série de transtornos nas regiões oeste e noroeste. Na região de Santo Ângelo, cinco municípios comunicaram a ocor- rência de vendavais à DCRS, sendo que três decretaram situação de emergência. Segundo o JCP (2006), o temporal provocou ventos de até $90 \mathrm{~km} / \mathrm{h}$ em Santo Ângelo, causando a queda de postes e de árvores e interrompendo o fornecimento de eletricidade. Em São Borja 70 casas foram destelhadas e praticamente toda a cidade ficou sem abastecimento de água, luz e telefone. Os ventos de mais de $100 \mathrm{~km} / \mathrm{h}$ afetaram, sobretudo, o Centro-Sul da cidade, levando o município a decretar situação de emergência.

Entre os dias 25 e $27 / 11$ os eventos $M$ e $N$ (TAB. 1) provocaram precipitação intensa e ventos fortes. Em São Borja o temporal provocou a morte de um menino de 12 anos. Na região Metropolitana as fortes chuvas e ventos de mais de $100 \mathrm{~km} / \mathrm{h}$ provocaram queda de árvores e de postes, destelhamento de casas e um acidente de trânsito com dois feridos graves em São Leopoldo. A falta de energia atingiu diversos pontos desse município. Em Caçapava do Sul, na região sul, o temporal danificou mais de 100 casas, deixando cerca de 500 pessoas desabrigadas. O município decretou situação de emergência em virtude dos prejuízos.

\subsubsection{Dezembro}

Dezembro foi o mês que registrou o maior número de eventos extremos associados à CCM (46), totalizando $50 \%$ das ocorrências no trimestre. Aproximadamente 10.300 pessoas foram atingidas em 46 municípios do Estado. As regiões de Santa Maria e Santo Ângelo foram as mais prejudicadas com $57 \% \mathrm{e}$ $28 \%$ das ocorrências, respectivamente.

Entre os dias 07 e 09/12/2003, o evento $O$ (TAB. 1) causou diversos transtornos na fronteira oeste, principalmente no município de Frederico Westphalen, sendo essa cidade uma das mais atingidas. $\mathrm{O}$ granizo danificou 560 residências segundo a prefeitura do município, após concluir o levantamento dos estragos, decretando situação de emergência. Em São Borja, na Fronteira-Oeste, em apenas uma hora, a estação meteorológica da Fundação Estadual de Pesquisa Agropecuária do RS (FEPAGRO) acusou precipitação de $42 \mathrm{~mm}$, o que representa um terço dos $126 \mathrm{~mm}$ 
esperados para o mês. Episódios de vendavais provocaram destelhamentos de casas e danos em lavouras nas regiões de Santo Ângelo e Santa Maria.

Os CCMs ocorridos entre os dias 12 e $15 / 12$ (eventos $P, Q, R$ e $S$; TAB. 1), foram responsáveis por uma série de destruições em todo o Estado. Esses eventos provocaram precipitação intensa, ocasionado alagamentos, enxurradas, enchentes, inundações e deslizamento em 34 municípios, conforme a DCRS (2006). Além disso, houve relato de um tornado na localidade de Santo Tomé (Argentina), fronteira com o município de São Borja (RS), onde as autoridades locais confirmaram que cinco pessoas morreram e 38 ficaram feridas na noite de 14/12. No município de São Miguel das Missões, no noroeste do RS, um raio matou uma criança em uma reserva indígena. Em São Ângelo foi registrado outro óbito em condições não informadas. O referido município foi atingido por vendaval e alagamentos em 09/12 e por enxurras em 15/ 12, ambos associados à CCM.

O excesso de precipitação, verificado entre os dias 12 e 15/12, causou os primeiros episódios de enchentes nesse período, desabrigando um grande número de famílias. No município de Santa Maria, área central do Estado, foi decretada situação de emergência, devido à chuva que atingiu a região. Segundo o setor de meteorologia da Base Aérea, somente no dia $15 / 12$, durante 16 horas, o volume de precipitação foi de $186 \mathrm{~mm}$, índice maior que o esperado para o referido mês.

Entre 20 e 22/12/2003, dois eventos de CCM ( $T$ e $U$; TAB. 1), causaram fortes chuvas na região da Fronteira-Oeste do Estado, agravando a situação dos municípios já atingidos pelas enchentes do rio Uruguai. A cidade de Uruguaiana decretou situação de emergência em função de um temporal ocorrido na madrugada do dia 22/12, o que acabou por agravar ainda mais a situação do município já atingido pelas cheias. Durante o evento $V$ (TAB. 1), ocorrido entre os dias 25 e 27/12, não foram verificadas ocorrências expressivas no RS, já que DCRS e o JCP não relataram nenhuma situação relacionada a desastres.

\section{CONCLUSÕES}

Os dados da DCRS e do JCP evidenciaram uma série de ocorrências de eventos meteorológicos adversos observados nos dias de ocorrência de CCM. Os resultados apontados nesse estudo corroboram a hipótese de que os CCMs normalmente estão associados a tempo severo. Em média, a cada quatro eventos, três causam algum tipo de desastre. Ao todo, 16.500 pessoas foram atingidas no RS, em virtude de desastres provocados pelos eventos no período de estudo. Além dos incontáveis prejuízos materiais, foram relatados casos de onze pessoas que perderam a vida, vitimadas por desastres associados aos CCMs. Em relação ao número de óbitos, a cada dois eventos, um produziu mortos e/ou feridos.

Devido à abrangência dos eventos de $\mathrm{CCM}$ e o grande número de desastres associados, torna-se muito difícil realizar um levantamento detalhado, identificando os prejuízos causados e o número exato de pessoas atingidas. Sabe-se ainda, que muitos outros casos não são noticiados nos meios de comunicação, nem relatados à Defesa Civil. Portanto, estima-se que uma parcela ainda maior de desastres, e conseqüentemente de pessoas atingidas, esteja associada à CCM. Sendo assim, é fundamental que os municípios informem detalhadamente à Defesa Civil os danos causados por fenômenos meteorológicos adversos, para que seja possível uma melhor avaliação dos impactos causados por esses eventos no Estado, possibilitando medidas de prevenção e assistência à população.

Acredita-se que o período de análise foi suficiente para avaliar o impacto de eventos de CCMs no RS e demonstrar a importância do seu estudo na gestão de desastres. Os prejuízos materiais e perdas de vidas humanas reportados nas datas de ocorrência de CCMs evidenciaram condições de risco na região. Isto é, a existência dos CCMs constitui-se numa ameaça de origem natural e a estrutura social do Estado do RS é particularmente vulnerável a este fenômeno.

Apesar dos CCMs estarem normalmente associados à condições adversas de tempo meteorológico, os eventos trazem, por sua vez, precipitações benéfi- 
cas, principalmente em função do período de ocorrência, ligado à época do plantio e desenvolvimento das duas maiores culturas do Estado: o milho e a soja. A compreensão da gênese, desenvolvimento, mecanismos de formação e comportamento dos CCMs são de vital importância para que o Estado do RS possa estar preparado para lidar com as adversidades decorrentes desses eventos e, ao mesmo tempo, consiga tirar proveito dos benefícios, nesse caso, a precipitação associada.

\section{AGRADECIMENTOS}

À equipe da Divisão de Satélites Ambientais (DSA) do Instituto Nacional de Pesquisas Espaciais (INPE) pela disponibilização dos dados do aplicativo ForTraCC e das imagens de satélite.

\section{REFERÊNCIAS}

ABDOULAEV S.; STAROSTIN, A.; CASARIN, D.P. Sistemas de mesoescala de precipitações no Rio Grande do Sul. Parte 1: Descrição Geral. IX Congresso Brasileiro de Meteorologia, SBMET. Campos de Jordão. Anais... 2, p. 936 - 940, 1996.

ALCÁNTARA-AYALA, I. Geomorphology, natural hazard, vulnerability and prevention of natural disasters developing countries. Geomorphology, v.47, p.107124, 2002.

AYSAN, Y.; OLIVER, P. Housing and Culture after Earthquakes. Oxford Polytechnic, Oxford, 1987.

BRYANT, E. A. Natural hazards. Cambridge University Press, Cambridge. 288 p., 1991.

CASTRO, A. L. C. Glossário de defesa civil: estudo de riscos e medicina de desastres. Brasília: MPO/ Departamento de Defesa Civil, 283 p., 1998.

. Manual de planejamento em Defesa Civil. Vol.1. Brasília: Ministério da Integração Nacional/Departamento de Defesa Civil, 133 p., 1999.

- Manual de Desastres: desastres. Brasília: Ministério da Integração Nacional, 174 p., 2003.
INSTITUTO NACIONAL DE PESQUISAS ESPACIAIS (CPTEC/INPE). Centro de Previsão do Tempo e Estudos Climáticos. Apresenta: imagens de satélite. 2004. Disponível em $<$ http://www.cptec.inpe.br $>$. Acesso em jul. 2004.

CUNY C.F. Disasters and Development. Oxford University Press. New York, Oxford, 1983.

DEFESA CIVIL DO RIO GRANDE DO SUL (DCRS). 2006. Apresenta: textos e tabelas. Disponível em: $<$ www.defesacivil.rs.gov.br $>$. Acesso em nov. 2006.

FRITSCH, J.M.; KANE, R.J.; CHELIUS, C.R. The Contribution of Mesoscale convective Weather Systems to the Warm-Season Precipitation in the United States. J Clim Appl Meteorol., v.25, p.1333 - 1345, 1986.

GLICKMAN, T. S. Glossary of meteorology. Boston: American Meteorological Society, 855 p., 2000.

GUEDES, R.L.; SILVA DIAS, M.A.F. Estudo de tempestades severas associadas com o jato subtropical na América do Sul. In: III CONGRESSO BRASILEIRO DE METEOROLOGIA, SBMET. Belo Horizonte, MG, 3-7 Dezembro, Anais..., v.1, p.289 - 296, 1984.

GUEDES, R.L. et al. Trajetórias dos sistemas convectivos sobre o continente americano. VIII CONGRESSO BRASILEIRO DE METEOROLOGIA, SBMET, Anais... 2, p.77 - 80, 1994.

GUIDICINI, G.; IWASA, O.Y. Ensaio de correlação entre pluviosidade e escorregamentos em meio tropical úmido. São Paulo: Instituto de Pesquisas Tecnológicas do Estado de São Paulo - IPT, 48 p. (Relatório n. 1080), 1976.

JORNAL CORREIO DO POVO (JCP). 2004. Apresenta: notícias, reportagens e matérias especiais. Disponível em $<$ www.correiodopovo.com.br $>$. Acesso em nov. 2006.

KNIGHT, C. A.; KNIGHT, N. C. Hailstorms. In: DOSWELL III, C. A. Severe convective storms. Boston: American Meteorological Society, 2001. Meteorological 
Monographs, v. 28, n. 50, p.223 - 249, 2001.

KOBIYAMA, M.; et al. Prevenção de desastres: conceitos básicos. Florianópolis: Ed. Organic Trading, 2006.

KULICOV, V. A.; RUDNEV, G. V. Agrometeorologia tropical. Havana: Científico-Técnica, 1980.

MACEDO, S.R.; VILA, D.; MACHADO, L.A. FORTRACC - Previsão a curto prazo e evolução dos sistemas convectivos. FORTRACC V1.1 - Guia de Usuário. São Paulo: INPE/CPTEC, 2004. Disponível em $<$ http://moara.cptec.inpe.br/pdf/FORTRACC.pdff $>$. Acesso em: 01/07/2006.

MACHADO, L.A.T. et al. Ciclo de vida de sistemas convectivos. In: VIII CONGRESSO BRASILEIRO DE METEOROLOGIA, SBMET, Anais... 2, p.323 - 326, 1994.

MACHADO, L.A.T. et al. Life Cycle Variations of Mesoscale Convective Systems over the Americas. Mon Weather Rev., v.126, p.1630 - 1654, 1998.

MADDOX, R.A. Mesoscale Convective Complexes. Bul Amer Meteorol Soc., v.61, n.11, p.1374 - 1387, 1980.

MADDOX, R.A. Large-Scale Meteorological Conditions Associated with Midlatitude, Mesoscale Convective Complexes. Mon Weather Rev., v.111, p.1475 - 1493, 1983.

MARENGO, J.A.; SOARES, W.R.; SAULO, C.; NICOLINI, M. Climatology of the Low-Level Jet East of the Andes as Derived from the NCEP-NCAR Reanalyses: Characteristics and Temporal Variability. J Clim., v.17, n.12, p.2261 - 2280, 2004.

MASKREY, A. El Riesgo. Capitulo 1 en: Navegando entre Brumas. La aplicación de los Sistemas de Información geográfica al análisis de riesgos em América Latina. Andrew Maskrey (Editor) ITDG/LA RED. Colombia, 1998. Disponível em $<\underline{\text { http:// }}$ www.desenredando.org/public/libros/1998/neb/ index.html>. Acesso em nov. 2007.
MCANELLY, R.L.; COTTON, W.R. The Precipitation Life Cycle of Mesoscale Convective Complexes over the Central United States. Mon Weather Rev., v.117, p. $784-808,1989$.

NOGUÉS-PAEGLE, J.; BERBERY, E.H. Low-level Jets over the Americas. Exchanges -Selected Research Papers. n 16, Jun/2000.

PAEGLE, J. Interactions between convective and largescale motions over Amazonia. In: The geophysiology of Amazonia: Vegetation and Climate Interactions. R. Dickerson ed., Wiley Intersciences, p.347 - 390, 1987.

SATYAMURTY, P.; NOBRE, C.A.; SILVA DIAS, P.L. South America. In: Meteorology of the Southern Hemisphere. Boston: A.M.S., v.27. p.119-139, 1998.

SCOLAR, J.; FIGUEIREDO, J.C. Análise das condições sinóticas associadas à formação de Complexos Convectivos de Mesoescala. In: VI CONGRESSO BRASILEIRO DE METEOROLOGIA, SBMET, Anais... v.2, p.457 - 461, 1990.

SELBY, M.J. Hillslope materials and processes. 2ed. Oxford: Oxford Univ. Press, 451p., 1993.

SEVERO, D.L.; GAN, M.A. Anomalias de precipitação na região sul do Brasil: resultados preliminares. In: XIIX Congresso Brasileiro de Meteorologia. Anais... SBMET, 2004.

SILVA DIAS, M.A.F. Sistemas de Mesoescala e Previsão de Tempo à Curto Prazo. Revista Brasileira de Meteorologia. v.2, p.133 - 150, 1987.

TORRES, J.C.; NICOLINI, M. A composite mesoscale convective systems over southern South America and its relationship to low-level jet events. VAMOS/CLIVAR/WRCP. Conference on South American low-level jet. Santa Cruz de la Sierra, Bolívia, Fevereiro/2002.

VELASCO, I.; FRITSCH, J.M. Mesoscale Convective Complexes in the Americas. J Geophys Res., v.92, n. D8, p.9591 - 9613, 1987. 
VIANA, D.R. et al. Mesoscale Convective Complexes in Rio Grande do Sul between October and December of 2003 and Associated Precipitation. Revista Brasileira de Meteorologia, 2009. No prelo.

VIANELLO, R. L; ALVES, A. R. Meteorologia básica e aplicações. Viçosa: UFV, 449 p., 1991.

WIJKMAN, A.; TIMBERLAKE, L. Desastres Naturales ¿Fuerza Mayor u Obra del Hombre? Earthscan, 1985.

WILCHES-CHAUX, G. La Vulnerabilidad Global. Capitulo 2 en: Los Desastres no son Naturales. Andrew Maskrey (Compilador). Colombia, 1993. Disponível em $<$ http://www.desenredando.org/public/libros/1993/ $\underline{\mathrm{ldnsn} / \mathrm{html} />}$. Acesso em nov. 2007. 